hep-th/0012081

SU-ITP-00/33

SLAC-PUB-8735

\title{
Closed strings from nothing
}

\author{
Matthew Kleban ${ }^{1}$, Albion Lawrence ${ }^{1,2}$ and Stephen Shenker ${ }^{1}$ \\ ${ }^{1}$ Department of Physics, Stanford University, Stanford, CA 94305 \\ ${ }^{2}$ SLAC Theory Group, MS 81, PO Box 4349, Stanford, CA 94309
}

\begin{abstract}
We study the physics of open strings in bosonic and type II string theories in the presence of unstable D-branes. When the potential energy of the open string tachyon is at its minimum, Sen has argued that only closed strings remain in the perturbative spectrum. We explore the scenario of Yi and of Bergman, Hori and Yi, who argue that the open string degrees of freedom are strongly coupled and disappear through confinement. We discuss arguments using open string field theory and worldsheet boundary RG flows, which seem to indicate otherwise. We then describe a solitonic excitation of the open string tachyon and gauge field with the charge and tension of a fundamental closed string. This requires a double scaling limit where the tachyon is taken to its minimal value and the electric field is taken to its maximum value. The resulting flux tube has an unconstrained spatial profile; and for large fundamental string charge, it appears to have light, weakly coupled open strings living in the core. We argue that the flux tube acquires a size or order $\alpha^{\prime}$ through sigma model and string coupling effects; and we argue that confinement effects make the light degrees of freedom heavy and strongly interacting.
\end{abstract}

December 2000 


\section{Introduction}

The known nonperturbative definitions of string- and M-theory vacua [1],2] are remarkable yet conceptually deficient. They provide unitary, holographic descriptions of quantum gravity; but locality is not manifest even at macroscopic scales. Any sort of background independence is also completely obscure. Experience tells us that the world is local down to microscopic scales. Certainly the systems described in [1,2] describe local spacetime physics; however, such physics is hard to extract from the field theoretic "boundary "variables used. It seems plausible that there should be another set of manifestly local "bulk" variables. Holography could then arise via the fixing of some large gauge symmetry of the theory [3]. Such a description would be a major step towards understanding holography in more general backgrounds.

A natural candidate for such a theory is string field theory; it is local down to the string scale, and it has a large gauge symmetry. However, closed string field theory, the natural guess for a theory of quantum gravity, does not seem to be complete as it stands. The state of the art [4] is an "effective" theory, defined perturbatively; vertices must be added at every order in $g_{s}$, and large regions of the moduli space of string diagrams are integrated over in the construction of these vertices. 1 There is no simple explanation within closed string field theory of the rapid divergence of closed string perturbation theory [6]; the effect must be contained in the new vertices. The physical D-branes which this divergence signals must be added to the theory by hand.

Open string field theory is in better shape. Bosonic open string field theory (and the NS sector of open superstring field theory) has a classical Lagrangian which can be written in closed form, and which generates all perturbative diagrams [0, 8, 9, 10]. Closed strings appear in intermediate channels of open string diagrams [11], and the open string theory covers the moduli space of closed string diagrams with at least one hole [8]. Perturbation theory diverges no more rapidly than field theory; again, this reflects the existence of D-branes, which contribute effects of order $e^{-1 / g_{\text {open }}^{2}}$ to scattering amplitudes.

It remains to find D-branes as open string field theory excitations, and to find a description of closed string theories in the absence of D-branes. The study of unstable D-branes, pioneered and largely carried out by A. Sen and his collaborators, provides an attractive answer for type II and bosonic string theories. Begin with unstable D-branes or D-brane-anti-D-brane pairs, filling all of space. These will contain open-string tachyons;

1 c.f. [5] for discussion of this issue with further references. 
the endpoint of tachyon condensation is the perturbative closed-string vacuum (up to closed-string tachyons) [12, 13, 14, 15, 16, 目 D-branes appear as configurations of the openstring tachyon field in the closed string vacuum; the open string vacuum remains at the core of the D-brane [21,22,23]. Such configurations have also been found using the tachyon potential calculated in open bosonic string field theory [24, 25, 26, 27, 28].

We would therefore like to describe type II string theory via open string field theory living on $N$ unstable D9-branes of type IIA, or on $N D 9-\bar{D} 9$-brane pairs in type IIB [29,30] (possibly as $N \rightarrow \infty$ [30]). This raises a number of questions, particularly: what are the dynamics of open strings in the closed string vacuum? How do closed strings emerge as weakly coupled excitations?

We will attack these questions by studying electric flux tubes with closed string charge as the tachyon condenses, as in [31,32, 27, 33, 34]. In section 2 we argue, following previous work, that that the open strings in the closed string vacuum are strongly coupled, at least in variables natural to world sheet conformal field theory. We will begin with a selective survey of the literature surrounding this issue. In particular our claim appears to contradict the worldsheet analysis of [35, 36, 37], and we discuss a resolution of this contradiction by pointing out order of limits implicit in various approaches. We will also address the rather different picture emerging from numerical calculations in the level truncation scheme 38,39 and discuss the possible relationship via nonlinear, nonlocal, field redefinitions. Our conclusion is that, at least in the variables natural to world sheet conformal field theory, the open strings should disappear through a confinement of the $U(1)$ gauge field on the unstable D-brane (or the diagonal $U(1)$ gauge field on a $D-\bar{D}$ pair) under which the string endpoints are charged; this picture was developed initially by P. Yi and his collaborators [31,32. In section 3 we study solutions of the tachyon-Born-Infeld action proposed in [13,40, 36, 41], and find a solution with the charge and tension of a macroscopic closed string. In this solution the the electric field must be scaled to its critical value (as in refs. [42,43]; see also [44]), and the profile appears completely unconstrained. We study the physics of this solution in Sec. 4, first discussing effects by which the flux tube may be localized, and next addressing the open string dynamics in the core of this flux tube. It appears that if the closed string charge is large, then the open strings in the core are light and weakly coupled. We argue that if the flux tubes are indeed localized, this will not be the case. The picture we arrive at is that closed strings are a collective excitation in a strongly-coupled open string background, following the scenario developed in [31,32].

2 Early work studying condensation of the open string tachyon can be found in [17, 18, 19, 20$]$. 


\title{
2. The fate of open strings, or the dynamics of nothing
}

\author{
"I ask for nothing, Master."
}

"And you shall receive it - in abundance."

- The Rocky Horror Picture Show

\subsection{Arguments for strongly coupled open strings}

Let us begin with an unstable Dp-brane in type II or bosonic string theory. The perturbative spectrum of open strings ending on the brane will include a tachyon, a $U(1)$ gauge field, massless scalars describing the transverse fluctuations of the Dp-brane, and a tower of massive open string modes. The unstable brane decays by condensation of the tachyon; at the minimum of the tachyon potential, the theory has only closed strings as perturbative excitations [12, 13, 14, 15, 16].

How do the open strings disappear as perturbative excitations? Sen [13] argued that the Lagrangian for open string modes vanishes at the endpoint of tachyon condensation, i.e.

$$
L=f(\phi) \tilde{L}(\{\Psi\})
$$

where $\phi$ is the tachyon, $f(\phi)$ vanishes at the minimum of the tachyon potential, and $\Psi$ represents the open string modes. In particular, worldsheet RG flow arguments 35, and calculations [36,45, 46, 47 using the background-independent string field theory proposed in [48], show that the action for the $U(1)$ gauge field with slowly varying field strength $F$ is as proposed in [13]:

$$
S=\int d^{p+1} x \frac{V(\phi)}{g_{s}} \sqrt{-\operatorname{det}\left(g_{\mu \nu}-F_{\mu \nu}\right)},
$$

where $V(\phi) / g_{s}$ is the potential energy for the tachyon and $V(\phi)=0$ in the closed string vacuum.

A related phenomenon is discussed in [49]. A nontrivial maximal rank, constant NSNS B-field in Euclidean space leads to a gauged $U(\infty)$ symmetry. The authors of [19] propose that the endpoint of tachyon condensation in this background is the "nothing state" for which the $U(\infty)$ symmetry is restored (the background is gauge-invariant). This symmetry forbids a nonvanishing gauge kinetic term. We adopt their coinage for our title.

A vanishing kinetic term generally signifies strong coupling; there is little cost in action for wild fluctuations over short distances in space. Said another way, we can rescale 
the fields to maintain a nonvanishing kinetic term, but then the interaction terms will have infinitely large coefficients. We expect the open strings to disappear via some sort of confinement mechanism as advocated in [31,32]. In particular the endpoints of the open strings are charged under the $U(1)$ gauge field in $(2.2)$. If the $U(1)$ confines, the the endpoints will be joined by an electric flux tube (see Fig. 1). In the following section we will argue that this flux tube is a piece of fundamental string, so that the open-string-plusflux-tube configuration is in fact a closed string [34].

unconfined electric flux

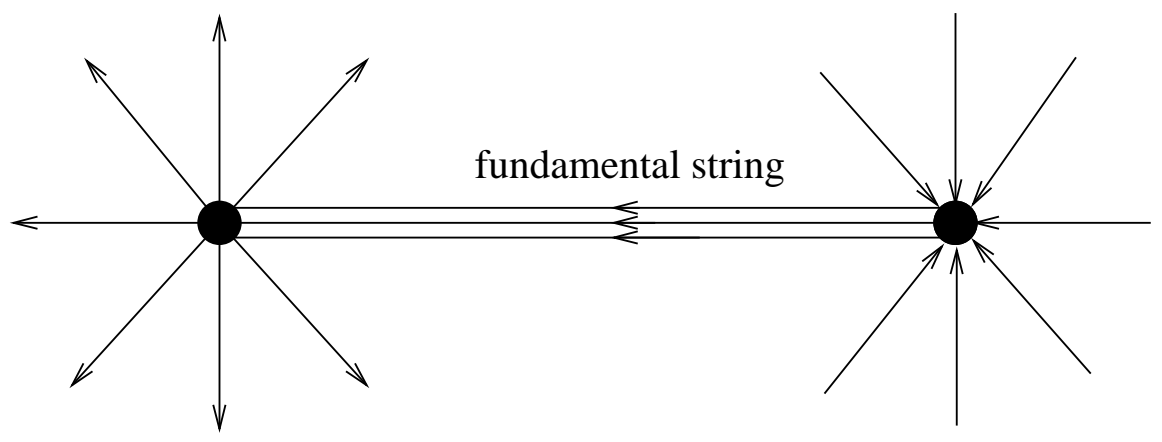

confined flux tube

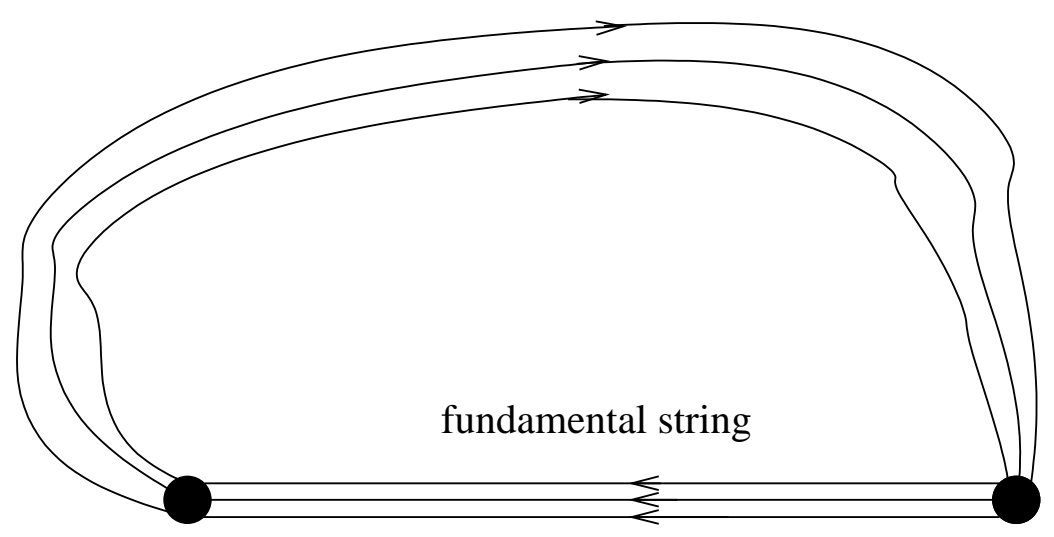

Figure 1. Confinement of an open string.

The tachyon is also strongly coupled near its minimum. 3 Assume for the moment that $F=0$ and choose coordinates on the field space of the tachyon for which the tachyon kinetic term is the Lagrangian is canonical:

$$
L_{k i n}=\frac{1}{g_{s}}(\partial \phi)^{2}
$$

3 We will persist in calling $\phi$ "the tachyon" even near the minimum of $V(\phi)$. 
The calculations in [50,40,36,41] show that near the closed string vacuum $\phi=0$,

$$
V(\phi)=-\frac{1}{g_{s}} \phi^{2} \ln \phi^{2}+\ldots
$$

This precise form was also found using a scalar field theory model of tachyon condensation in [51,52. (This is exact for the bosonic string but has corrections for the superstring, as one can see by performing the appropriate field redefinition in [52,41].) Since the second derivative of this potential diverges logarithmically at $\phi=0$, it is tempting to claim that $\phi$ simply decouples [40]. However, the higher derivatives diverge as increasing inverse powers of $\phi$. In any Feynman diagram describing tachyon scattering near $\phi=0$, the diverging vertices overwhelm the vanishing propagators for any ratio of propagators to vertices: a power law divergence always overwhelms zeros from arbitrary powers of inverse logs.

The mechanism proposed in [13] is to set $V(\phi)=0$ and treat the gauge fields as Lagrange multipliers. Upon integrating them out, the charged states will be removed. This seems simpler than the strong coupling scenario, but really is much the same. In field theory, treating the gauge fields as Lagrange multipliers is the lowest order approximation in a strong-coupling expansion. Despite the simplicity of this lowest-order solution, one still understands the gauge fields as fluctuating wildly. Furthermore, $\phi$ will fluctuate for any finite $g_{s}$; and classical excitations of $\phi$ describe D-branes and should be included in the theory. In the end, we will have to face the full complications of a strongly coupled system.

\subsection{Worldsheet analysis}

Equations (2.1) and (2.2) follow from world sheet and related string field theory analyses [35,40,36, 41]. These works study tachyon condensation by adding a relevant boundary operator

$$
\begin{aligned}
& S=S_{0}+\delta S \\
& S_{0}=\int_{D} d^{2} \sigma(\partial X)^{2} \\
& \delta S=\oint_{\partial D} d t T(X(t))
\end{aligned}
$$

to the tree-level open string worldsheet (a disc with circumference $2 \pi$ ) and studying the infrared fixed point of the induced RG flow. The background-independent open string field theory or "boundary string field theory" (BSFT) 48,50,53,54,55 constructs the effective action using the worldsheet beta function and the perturbed two-point functions. The 
result is that the effective action at the fixed points of these flows is the boundary entropy as defined in [56].

Various choices of $T$ describe different endpoints. The perturbation

$$
T(X)=\lambda \sin (k \cdot X)
$$

(for $k^{2}<1$ ) studied in [35] is integrable, and the flow can be studied using Bethe-ansätz techniques [57]. It describes an array of lower-dimensional D-branes, or a D-brane on a circle, in the IR limit $\lambda \rightarrow \infty$. Similarly, a Gaussian perturbation

$$
T(X)=u X^{2}
$$

as studied in [40,36] describes a single D-brane in the IR limit $u \rightarrow \infty$. (More general quadratic interactions are discussed in [53].) We note in passing that the tachyon profile describing the D-branes is infinitely thin, contradicting our expectations from the lowenergy effective action as well as explicit calculations [24,25,26 using an approximation to Witten's cubic string field theory, but in accord with our physical expectations [58]. The calculations in [36] demonstrate that the thinness of the D-brane arises from higherderivative corrections which are not fully taken into account in the low energy effective actions arising from in either version of open string field theory. This is an example of the sensitivity of results about string scale dynamics to truncation and field redefinition.

Finally, the constant perturbation

$$
T(X)=\frac{a}{2 \pi}
$$

describes the closed string vacuum in the IR limit $a \rightarrow \infty$. Note that in these variables,

$$
\phi \propto e^{-a / 2}
$$

in the bosonic string for a constant tachyon [40,36]. A similar field redefinition in terms of error functions can be performed for the superstring [41.

In the case of the constant perturbation (2.7), the terms in the tree-level effective action up to two derivatives scale with the tachyon as $V(\phi)$ near the closed string vacuum. As we discussed above, the vanishing of the kinetic terms signals strong coupling. The interaction terms also vanish. But in the field theory limit of open string diagrams, the

4 While higher derivative terms scale to zero more quickly - c.f. sec. 5 of [36]. 
vertices will vanish but the propagators will diverge. We expect the effective open string coupling to diverge; roughly,

$$
g_{e f f}^{2}=\frac{g_{s}}{V(\phi)} .
$$

Two aspects of the discussion in [35, 36] are apparently at odds with our strongcoupling scenario. First, one may study higher-loop amplitudes in the presence of the perturbation (2.7). The authors of [36] argued that since the perturbation is a constant, one might expect that each hole is weighted with:

$$
\tilde{g}_{e f f}^{2} \sim e^{-\oint a}=e^{-2 \pi a}
$$

As $a \rightarrow \infty$ the holes will vanish, so the effective open string coupling would be weak.

However we expect that on general grounds the correct normalization of propagators and vertices is given by the effective action (2.1), (2.2) extracted from world sheet results. One can already see at the level of the four-point function that the integration over moduli of the worldsheet will give a scaling different from that implied by (2.10). For example, a representation of four-string scattering is given by sewing together cubic vertices and propagators using the cubic string field theory in [7]. The relation would be a conformal mapping along the lines of [59]: when two vertex operators collide on the disc, the propagator in the cubic SFT picture is getting long. The constant tachyon perturbation is not invariant under this conformal transformation; and in the limit that the propagator is of length $L$, the amplitude will not be suppressed by $e^{-a L}$. A Riemann surface picture of the vertices and propagators in BSFT has not yet been developed; when it has, the scaling with $a$ of vertices and propagators should be consistent with the effective action (2.2).

A more serious objection to the strong coupling picture is that the RG flows induced by the perturbations (2.5)-(2.7) are nonsingular; there is no divergence or bad behaviour at the level of the disc which would signal strong coupling. The disc-level RG calculations seem to give the right physics. For perturbations (2.5) and (2.6) describing D-branes at the IR fixed points, the open strings are bound by the perturbing potential to the Dbranes, and the D-brane tension is given exactly by the tree-level boundary entropy. For the constant perturbation (2.7), the effective action for the open strings simply disappears.

Nonetheless, we still see no contradiction with the strong-coupling scenario. To begin with, there is no reason for the emergence of strong coupling to appear as a divergence on the disc. For example, the worldsheet theory of perturbative type II string theory in ten dimensions is not singular as the dilaton is dialed to strong coupling; rather, string 
diagrams get larger at each order in perturbation theory. Similarly, the linear dilaton vacuum is an exact CFT even though it contains a strong coupling region.

Secondly, if we take our effective action seriously, at any value of $\phi$ away from zero, we can scale $g_{s}$ to be much smaller that $V(\phi)$. Thus, the disc amplitude accurately describes physics arbitrarily close to the closed string vacuum for arbitrarily small $g_{s}$.

Finally, when D-branes are present, we may self-consistently allow the strings to end only on the D-branes. The boundary entropy as measured by such worldsheets must be the D-brane tension as proven in [60,61]. The mystery is why the strings seem to be bound to the D-branes at tree level, when the strong coupling picture suggests that the disappearance of open strings far from the D-brane is described by some sort of confinement mechanism. For macroscopic strings, a likely answer is that the effective inverse coupling $V(\phi) / g_{s}$ can be treated as a dielectric constant for the $U(1)$ gauge field, as in [62]. The endpoints of strings are charged and will energetically prefer to live in a region with large dielectric constant. Far from the D-branes there is no gradient of the tachyon field; but the wavefunction for the string endpoints will generally have support intersecting the Dbrane. When the D-branes are absent, we must resort to the strong coupling picture to rid ourselves of open strings.

\subsection{Numerical calculations using cubic SFT}

The present excitement surrounding open string field theory was in large measure triggered by a calculation of the tension of an unstable D-brane [15] using the open string field theory of [7] combined with the level truncation scheme developed in [63]. Further work on the low-energy effective action for string fields in this scheme appears in [64, 38, 39. The results of these calculations are again at odds with the strong coupling picture, and even with the effective action (2.2).

Before addressing these apparent contradictions, we should note that the RG calculations of [35,40, 36] are exact and include all of the $\alpha^{\prime}$ corrections; and while the level truncation calculations converge there is no understanding of why they do.

That said, the calculation of the tachyon potential energy [15, 16] is classical and gives the correct answer for the D-brane tension to high accuracy. If the open strings are strongly coupled in the closed string vacuum, why were we able to trust the calculation close to minimum of the tachyon potential? This problem is identical to that of the calculation of the D-brane tension in BSFT. Again, there exists an order of limits such that at any point 
in field space arbitrarily close to the minimum of the tachyon potential, one may dial the string coupling to be sufficiently small so that the effective coupling $V(\phi) / g_{s}$ is weak.

An apparently genuine disagreement with our scenario and with the form (2.2) of the effective action arises from calculations of the effective action for the tachyon and $U(1)$ gauge field, using the string field theory of [48]. At finite order in the level truncation expansion, the gauge field acquires a mass [64,38,39] indicating that gauge invariance is broken and the effective action (2.2) is incorrect. Furthermore there is no indication as yet that the string fields are strongly coupled. Results for the coefficient of the gauge field kinetic term have appeared in [38]; and while this coefficient is decreasing at each order in the level truncation approximation, it is not decreasing very rapidly.

Nonetheless, we expect the cubic SFT and BSFT to describe the same theory, using very different coordinates on field space. In the open string vacuum, there are known finite field redefinitions [65,38, 39, 36] which relate the fields used in cubic SFT to fields with standard gauge transformations as would appear in (2.2). In Appendix A we show that the field redefinition between these two versions of open SFT are singular in the closed string vacuum. We also show that if the effective action in BSFT variables is (2.2), then near the closed string vacuum the field redefinition will lead to large interaction terms in the action in the cubic SFT variables. We should also note that the field redefinitions could be highly nonlocal. This is already apparent near the open string vacuum as shown in Appendix A of [36].

Since the field redefinitions are singular at the closed string vacuum, there may be a preferred set of coordinates there in which physics is weakly coupled. Sen has advocated that the open string degrees of freedom are like angular variables at the origin in polar coordinates [66]. At this point the reader may ask why we should describe the closed string vacuum via open string field theory; the natural physical fields in the closed string vacuum are, after all, closed strings. As before, we emphasize that open SFT is the best candidate for a fundamental description of the theory. It is certainly not excluded that a weakly coupled open string description is valid in the physical vacuum, with some as yet poorly understood classical mechanism responsible for the removal of open string states from the spectrum.

Given the rapid convergence of numerical results, the results in [64, 38, 39] certainly deserve further study. But we see no contradiction yet with the picture developed in [13,40,36]; so we will continue to assume that this latter picture of tachyon condensation is correct, although it may not be the only picture. 


\section{Electric flux tube solutions}

As promised, we will search for an excitation of open string fields around the closed string vacuum, with the mass and charge of a fundamental string. An obvious obstacle confronts us-solitons typically have mass $\sim m_{s} / g_{\text {open }}^{2}$ while we seek a solution with mass $\sim$ $m_{s}$. We shall see, following previous work, how to resolve this.

We will study an unstable non-BPS brane in either type II or bosonic string theory. We will focus on the $U(1)$ gauge field $A_{\mu}$ and the neutral scalar "tachyon" $\phi$. There is no good reason to ignore the higher open string oscillator modes; indeed we will argue that such modes become light. These should appear via higher-derivative corrections to the Lagrangian we write down.

\subsection{The action}

For slowly varying $U(1)$ fields, we assume following [67,13] that the kinetic term is constructed with the open-string metric. We use the following form:

$$
S_{\phi, k i n}=\frac{1}{g_{s}} \int d^{p+1} x \sqrt{-\operatorname{det}\left(g_{\mu \nu}+\mathcal{F}_{\mu \nu}\right)}\left(-G_{S}^{\mu \nu} \partial_{\mu} \phi \partial_{\nu} \phi-V(\phi)\right) .
$$

Here $\mathcal{F}=2 \pi \alpha^{\prime} F-B^{\mathrm{NS}-\mathrm{NS}}$, and $G_{S, \mu \nu}$ is the symmetric part of $\left(g_{\mu \nu}+\mathcal{F}_{\mu \nu}\right)^{-1}$. We use the tachyon lagrangian computed in [50,40,36, 41], choosing field variables for which the tachyon kinetic term is canonical; $V(\phi)$ has the form (2.3) near $\phi=0$.

\subsection{General cylindrically symmetric solution}

We will work in cylindrical coordinates $(t, z, \rho, \Omega)$ in $p+1$ dimensions, where $z$ is the axial direction, $\rho$ the radial direction, and $\Omega$ the the $(p-2)$ remaining angular coordinates. We will look for a purely electric, axially symmetric solution: $\vec{E}=E(\rho) \hat{z}, F_{i j}=0$ for $0<(i, j) \leq p$; and $\phi=\phi(\rho)$.

Using our cylindrically symmetric ansätz in the action (3.1), we find that:

$$
S=-\frac{1}{g_{s}} \int d^{p+1} x \sqrt{1-\left(2 \pi \alpha^{\prime} E\right)^{2}}\left(V(\phi)+\left(\partial_{\rho} \phi\right)^{2}\right)
$$

The canonical momentum for the electric field is:

$$
D_{z}=\frac{\left(2 \pi \alpha^{\prime}\right)^{2} E}{g_{s} \sqrt{1-\left(2 \pi \alpha^{\prime} E\right)^{2}}}\left(V(\phi)+\left(\partial_{\rho} \phi\right)^{2}\right)
$$


and the Hamiltonian as a function of $E$ is:

$$
H=\int d^{p} x \frac{1}{g_{s} \sqrt{1-\left(2 \pi \alpha^{\prime} E\right)^{2}}}\left(V(\phi)+\left(\partial_{\rho} \phi\right)^{2}\right)=\int d^{p} x \frac{D}{\left(2 \pi \alpha^{\prime}\right)^{2} E} .
$$

We impose the charge quantization condition:

$$
Q=\int \rho^{p-2} d \rho D=k
$$

If we wish the tension to be $\frac{k}{2 \pi \alpha^{\prime}}$ as it must be for $k$ fundamental strings, then eq. (3.4) means that we must scale $2 \pi \alpha^{\prime} E=1$, which is its maximal value 68 . This limit allows us to have a very light soliton.

We can find a solution by varying the Lagrangian plus the charge constraint:

$$
\delta[S+\lambda(Q-k)]
$$

where $\lambda$ is a Lagrange multiplier. Varying with respect to $E$, we find that $E$ is a function of $\lambda$ and is therefore a constant. $\gamma=\sqrt{1-2 \pi \alpha^{\prime} E}$ scales out of eq. (3.2), so that the equation of motion for $\phi$ is independent of $E$.

\subsection{Two solutions}

Flux tube with tachyon profile

Following [24,25,26,27,28, we assume that eq. (3.2) has a cylindrically symmetric lump solution. Even without a specific solution, we can study the energetics by scaling arguments. The tachyon potential has been computed at tree level, so the only dimensionful parameter in the solution will be $\alpha^{\prime}$ which we set to one. The transverse integral

$$
\beta=\int \rho^{p-2} d \rho\left(V(\phi)+\left(\partial_{\rho} \phi\right)^{2}\right)
$$

will be order one in these units (possibly times some integer $n$ if there are several lumps). We can solve eq. (3.5) for $E$ in terms of $\beta, g_{s}$. The energy per unit length (along $z$ ) of the solution is:

$$
H=\frac{1}{g_{s}} \sqrt{\beta^{2}+g_{s}^{2} k^{2}}
$$

which is the tension of the bound state of a D-string and $k$ fundamental strings. This should come as no surprise since it is believed [24,25,26, 27,28] that lump solutions of the tachyon should correspond to D-branes. 
If we expand the Born-Infeld action to quadratic order in $F$, we recover an action almost identical to that discussed in [62] as a model of confinement. $5(\phi)$ appears as a field-dependent dielectric constant. Although $E$ is constant everywhere, the canonical field momentum is proportional to $V$ and dies off away from the flux tube; thus the energy and fundamental string charge are finite.

\section{Fundamental string solution}

It is clear from eq. (3.4) that if we wish a solution with fundamental string tension, we must work with the full Born-Infeld action in the limit $\gamma \rightarrow 0$. In this limit, we must also scale the tachyon to keep $H$ and $\vec{D}$ finite. We will take the double scaling limit:

$$
\begin{aligned}
\sqrt{1-\left(2 \pi \alpha^{\prime} E\right)^{2}} & \sim-\frac{\epsilon^{2} \ln \epsilon^{2}}{g_{s} k} \\
\phi & \sim \epsilon g(\rho) .
\end{aligned}
$$

As $\epsilon \rightarrow 0$, the tachyon equations of motion vanish identically. $g$ is constrained only by charge quantization:

$$
\int \rho^{p-2} d \rho g^{2}+\left(\partial_{\rho} g\right)^{2} / \ln \epsilon^{2}=1
$$

Thus there is a continuous infinity of flux tube configurations, as we will discuss below. These results are implicit in refs. [32,27].

\section{Physics of the Scaling Limit}

In order to study the properties of open string fields, we will study the action for a generic open string scalar field $\chi$. Following the arguments in 667,13, 36] we use the Lagrangian

$$
S=\frac{1}{g_{s}} \int d^{p+1} x V(\phi) \sqrt{-\operatorname{det}\left(g_{\mu \nu}+F_{\mu \nu}\right)}\left(G_{S}^{\mu \nu} \partial_{\mu} \chi \partial_{\nu} \chi-m^{2} \chi^{2}+\lambda \chi^{3}+\ldots\right) .
$$

Here $m^{2} \sim M_{s}^{2}$ and $\lambda \sim 1$. If we scale $g_{s}$ out of the kinetic term, the cubic coupling will be proportional to the open string coupling $g_{\text {open }}=\sqrt{g_{s}}$.

5 The difference is that in that work, the function of $\phi$ multiplying $F^{2}$ differed from $V(\phi)$ in such a way that electric flux tubes emanating from a point source were energetically preferred to a spherically symmetric configuration of the electric field emanating from the same source. This is not guaranteed by the Maxwell limit of the Lagrangian at hand. 
In order to study the physics of open string fluctuations around our flux tube configuration, we expand (4.1) in small fluctuations, and rescale the fields and coordinates so that the kinetic terms are all of order 1 as $\epsilon \rightarrow 0$ in eq. (3.9). The effective mass and coupling of the string theory will appear as the coefficient of quadratic and higher-order terms in the effective action for the string modes. In particular, if interaction terms become large then we can conclude that the system is strongly coupled.

In the open string vacuum, we know that the limit $2 \pi \alpha^{\prime} E \rightarrow 1$ will send the open string coupling to zero [68 42] 43. On the other hand, if we scale the tachyon we expect the effective coupling to be $g_{\text {eff }}^{2}=g_{s} / V(\phi)$. These two effects compete in the double scaling limit.

\subsection{The open string vacuum}

To check our formalism and as a warm up, we will first expand the action around the open string vacuum in the limite $2 \pi \alpha^{\prime} E \rightarrow 1$. We define $\phi$ so that $V(\phi)=1-\phi^{2}+\ldots$ and we scale $\phi$ to zero independently of $1-\left(2 \pi \alpha^{\prime} E\right)^{2}$.

As before we scale

$$
1-\left(2 \pi \alpha^{\prime} E\right)^{2} \sim \epsilon^{4}
$$

In this limit, the Lagrangian for $\chi$ is:

$$
S=\frac{1}{g_{s}} \int d t d z d^{p-1} x\left(\frac{1}{\epsilon^{2}}\left[\left(\partial_{t} \chi\right)^{2}-\left(\partial_{z} \chi\right)^{2}\right]-\epsilon^{2}\left[(\vec{\nabla} \chi)^{2}+m^{2} \chi^{2}+\lambda \chi^{3}\right]\right)
$$

where we have suppressed finite constants (factors of $2 \pi$ and so on).

We wish to rescale $\chi$ and the coordinates $t, z, \vec{x}$, so that the kinetic terms are independent of $\epsilon$. We write the most general rescalings:

$$
\begin{aligned}
& \chi=\epsilon^{\delta} \tilde{\chi} \\
& (t, z)=\epsilon^{\beta}(\tilde{t}, \tilde{z}) \\
& \left(x_{1}, \ldots, x_{p-1}\right)=\epsilon^{\beta+2}\left(\tilde{x}_{1}, \ldots, \tilde{x}^{p-1}\right)
\end{aligned}
$$

The scaling of $\vec{x}$ differs from that of $t$ and $z$ due to the anisotropy of the open string metric.

A canonical ( $\epsilon$-independent) kinetic term requires:

$$
\delta=1-\frac{p-1}{2}(\beta+2)
$$

$\beta$ is not yet fixed; it rescales the time coordinate and thus the energy scales. We set $\beta=0$, thus measuring energy in units of $M_{s}$. 
We have simply reproduced the NCOS scaling. The scalar mass term scales as:

$$
m^{2} \epsilon^{4} \tilde{\chi}^{2}
$$

if $m \sim M_{s}$, then the mass in (4.5) is of order $M_{s, e f f} \sim \epsilon^{2}$ as in NCOS theory. The cubic term in $\chi$ scales as:

$$
\tilde{\lambda} \tilde{\chi}^{3} \sim \epsilon^{6-p} \tilde{\chi}^{3}
$$

The coupling has mass dimension $(5-p) / 2$. To find the dimensionless coupling, we should

multiply this by $M_{s, e f f}^{(p-5) / 2}=\epsilon^{p-5}$, as $M_{s, e f f}$ is the natural mass scale in the theory. The dimensionless coupling

$$
\tilde{\lambda} M_{s, e f f}^{(p-5) / 2} \sim \epsilon
$$

which is the expected scaling for the open string coupling [68,42, 43]. Finally, if we write the line element for the open string metric

$$
d s^{2}=G_{\mu \nu}^{o p e n} d x^{\mu} d x^{\nu}
$$

and rescale the coordinates so that:

$$
d s^{2}=\left(\alpha_{e f f}^{\prime}\right) \eta_{\mu \nu} d \tilde{x}^{\mu} d \tilde{x}^{\nu}
$$

the the rescaling is precisely that in (4.3).

\subsection{The fundamental string solution}

For the solution with $k$ fundamental strings in the closed string vacuum, the scaling (3.9) of the tachyon potential keeps the dimensionless coupling of the $\chi$ finite. In this scaling limit, the action (4.1) is:

$$
S=\int d^{p+1} x g(\rho)\left(k\left[\left(\partial_{t} \chi\right)^{2}-\left(\partial_{z} \chi\right)^{2}\right]-k\left(\frac{-\epsilon^{2} \ln \epsilon^{2}}{g_{s} k}\right)^{2}\left[(\vec{\nabla} \chi)^{2}+m^{2} \chi^{2}+\lambda \chi^{3}+\ldots\right]\right),
$$

where we have ignored the subleading $\epsilon^{2} g^{2} \ln g^{2}$ term in $V(\epsilon g)$. First, in order to make the kinetic term isotropic, we rescale $\vec{x}$ :

$$
\vec{x}=\frac{-\epsilon^{2} \ln \epsilon^{2}}{g_{s} k} \overrightarrow{\tilde{x}} .
$$


Next, in order to make the kinetic terms canonical, we rescale $\chi$ :

$$
\chi=\left(\frac{-\epsilon \ln \epsilon}{g_{s} k}\right)^{(1-p) / 2} \frac{1}{\sqrt{g(\rho) k}} \tilde{\chi} .
$$

where we have assumed $g(\rho)$ varies slowly.

In this limit the mass still scales as $\sqrt{1-\left(2 \pi \alpha^{\prime} E\right)^{2}}$ :

$$
S_{m a s s} \sim \int d^{p+1} \tilde{x} \frac{-\epsilon^{2} \ln \epsilon^{2}}{g_{s} k} m^{2} \tilde{\chi}^{2}=\int d^{p+1} \tilde{x}\left(M_{e f f}^{\prime}\right)^{2} \tilde{\chi}^{2} .
$$

The cubic term scales as:

$$
S_{c u b i c} \sim \int d^{p+1} \tilde{x} \frac{1}{\sqrt{g(\rho) k}}\left(\frac{-\epsilon^{2} \ln \epsilon^{2}}{g_{s} k}\right)^{(5-p) / 2} \tilde{\chi}^{3}=\int d^{p+1} \tilde{x} \tilde{\lambda} \tilde{\chi}^{3}
$$

The dimensionless coupling scales as:

$$
G_{\text {open }} \sim \tilde{\lambda}\left(M_{e f f}^{\prime}\right)^{(p-5) / 2}=\frac{1}{\sqrt{g(\rho) k}}
$$

Recall that $\int d^{p-1} \vec{x} g=1$. Thus if the flux tube has transverse size $l_{s}, g \sim 1$ inside the flux tube and the open string modes are weakly coupled at large $k$. Note that $G_{\text {open }}$ does not depend on $g_{s}$. Outside the flux tube, $g$ vanishes and the background is truly strongly coupled.

We are now left with two puzzles. First, can we remove the continuous infinity of possible profiles? Secondly, for $k \gg 1$ fundamental strings, how do we explain the apparent appearance of light, weakly coupled open string degrees of freedom?

\subsection{Localization of flux tubes}

The apparent degeneracy of flux tube profiles is serious, as such degrees of freedom do not exist for the fundamental string we are attempting to describe.6 However, the solution above is naturally subject to both sigma model corrections and quantum corrections. Some combination of these two could localize the flux tube.

One might think that the natural scale at which sigma model corrections (namely, corrections from higher-derivative terms in $\phi$ and $F$ ) would become important is $\ell_{s, e f f}$. If we assume cylindrical symmetry, however, this is not the case: the characteristic scale of

6 Additional discussion of this issue can be found in refs. [27,34]. 
transverse spatial fluctuations is still $\alpha^{\prime}$. One can see this by inserting a solution $\phi=\epsilon g(\rho)$ into some higher-derivative term and comparing it to the kinetic energy. Only terms with $\rho$ derivatives will contribute. The open string metric in the $\rho$ direction does not scale with $\sqrt{1-\left(2 \pi \alpha^{\prime} E\right)^{2}}$, so the derivative expansion for these terms is an expansion in $\alpha^{\prime}$.

The other option, explored in [32], is that nonperturbative corrections arising from $D(p-2)$-brane condensation localize the flux tube. The claim is that this condensation confines the open string degrees of freedom, and any flux tube has a width naturally associated with the confinement length. In [32] such an effect was argued for $D 2-\bar{D} 2$ pairs as arising from D0-brane instantons stretching between the two D2-branes. In this case the flux tube appears infinitely thin. Given the above discussion of $\alpha^{\prime}$ corrections, it is plausible that such corrections smear out the solution out to width $\alpha^{\prime}$.

The authors of [32] similarly argue for such an effect in $D 3-\bar{D} 3$ systems from type IIB S-duality. Here D-strings stretching between the D3-branes are monopoles magnetically charged under the diagonal $U(1)$. One may use S-duality to argue that these condense and confine electric flux, but the string coupling is strong in the S-dual picture so this description is heuristic at best.

In the end our belief is that some combination of strong quantum effects and $\alpha^{\prime}$ corrections should lead to a flux tube profile with width of order $\alpha^{\prime}$. Clearly our understanding is unsatisfactory and the subject deserves further study.

\subsection{Weakly coupled open strings}

The results for the open string spectrum and coupling in the core of the flux tube are very confusing. If the naive estimates are correct and the flux tube indeed corresponds to $k$ fundamental strings, then for $k \gg 1$ there should be a Hagedorn density of light, weakly coupled excitations in addition to the spatial fluctuations of the fundamental strings (which are captured by the collective coordinates of the flux tube). In particular these additional weakly coupled states would affect the results of perturbative closed string scattering, even at low energies.

If the flux tubes are spread out over a transverse volume of order $k \ell_{s}^{p-1}$, then the effective coupling $1 / \sqrt{k g(\rho)}$ is order one or higher and it is plausible the open strings disappear from the spectrum in the core of the tube. If the flux tube is small, of order $\ell_{s}$ in size, then the Compton wavelength of the light open string modes is much larger than 
the width of the flux tube by a factor of $1 / \epsilon^{2}$. These light modes prefer to stay within the flux tube, but this costs an uncertainty principle energy of order

$$
M_{s} \sim M_{s, e f f} / \epsilon^{2} \gg M_{s, e f f}
$$

So these light excitations are pushed to energies much higher than their characteristic string scale. Since the coupling is of order $1 / \sqrt{k}, M_{s}$ is nonperturbatively larger than $M_{s, e f f}$ if $\epsilon \ll 1$, and there is no reason to assume the modes at energies $M_{s}$ are weakly coupled.

However, quantum fluctuations generally keep us from setting $\phi$ strictly to its minimum; this would be reflected in a minimal value for the scaling parameter $\epsilon$. Nonetheless, we can see that $\epsilon$ will go to zero in the limit of vanishing $g_{s}$. Upon examining the Schrödinger equation for the zero mode $\phi_{0}$ of $\phi$, it is clear that the wavefunction of the ground state should scale as $g_{s}^{1 / 2}\left(\ln g_{s}\right)^{\delta}$. If we assume this is a minimum value for $\phi$ and plug this into $V(\phi)$, we find that the minimum value for $\epsilon$ is

$$
g_{s}=\epsilon^{2} f(\ln \epsilon)
$$

And so, up to logarithms,

$$
\ell_{s}=g_{s} \ell_{s, e f f}
$$

Thus, for sufficiently small $g_{s}$ the energies of the of the localized light modes in the flux tube are nonperturbatively larger than their masses because the effective open string coupling is of order $1 / \sqrt{k}$. At such energies there is no longer any reason to assume that the open strings interact weakly.

At any rate, while we have unfortunately placed the open strings out of any calculational control, we no longer see any paradoxes arising from our flux tube solution and so from our strong-coupling picture of open string dynamics in the closed string vacuum.

\section{Conclusions}

To take stock, let us consider the pros and cons of studying closed strings as solitons in tachyon condensed open string theory (as opposed to keeping explicit closed string degrees of freedom).

First, the pros: in contrast to closed-string field theory, open-string field theory may be nonperturbatively well defined, as well as bulk gauge covariant. Within this framework, 
the strongly coupled, confining picture introduced in [31,32] provides a natural explanation for the vanishing of typical open string states after tachyon condensation [32]. Begin with a rather large open string when the tachyon vev is in the open string vacuum. The open string state consists of two opposite charges joined by a section of fundamental string, with unconfined flux flowing off the charged ends. As the tachyon rolls, the $U(1)$ becomes confining, and the flux squeezes into a flux tube connecting the two charged ends. But in our scaling limit, the fundamental string has the same tension and charge as the flux tube, and so one really has a closed loop of flux, i.e., a closed string stated.

Now the cons. Basically nothing is calculable; the degrees of freedom are strongly coupled, and stringy rather than field theoretic (in contrast to the background-dependent descriptions of [1,2]). Such a messy description almost cries out for new, more manageable variables. Sen has made an intriguing analogy to polar coordinates [66. If we take a field theory with two real scalar fields, we can rewrite the theory in polar coordinates on field space:

$$
S=\frac{1}{\hbar} \int d^{d} x\left[(\partial r)^{2}+r^{2}(\partial \theta)^{2}\right]
$$

$\theta$ gets strongly coupled near the origin, but the Cartesian coordinates are still weakly coupled at small $\hbar$. In the present case, the perturbative excitations at $V(\phi)=0$ are closed strings. Unfortunately their nonperturbative dynamics is problematic. Still, one might expect by this analogy that any description using explicit open string fields - for example either the cubic or the background independent SFT - would be analogous to the angular variables in polar coordinates, since the closed string states must disappear. Certainly the kinetic terms appear to vanish in the closed string vacuum for string fields fields in BSFT. We should note that it is still possible that the cubic SFT variables are well behaved at $V(\phi)=0$. Although the coefficients of the gauge kinietic term are decreasing at the lowest few orders of the level truncation expansion [38], they are not converging as quickly as the results for the D-brane tension [15], and there is no indication as yet that they vanish [38.70]. If the kinetic terms and the mass of the gauge field are of order one in units of $M_{s}$, and if the interaction terms are finite, then the variables natural to the cubic SFT will be perfectly good. One still requires an explanation of the disappearance of perturbative open string fields despite the finite mass and kinetic terms. One possibility is that the poles disappear due to higher-derivative terms, as occurs at low orders in the level truncation scheme [63] and in the field theory of the p-adic string [71].

7 Ashoke Sen has discussed similar picture [34, 69] 
To summarize: we have examined the construction of macroscopic closed strings from open string degrees of freedom, an excitations of the closed string vacuum. It appears that such a construction is consistent with the known physics of closed strings, but we still have no computational control over such solutions.

There are a number of issues that deserve to addressed at this point. First, our flux tube solutions are highly quantum-mechanical. A typical classical field configuration has field strengths of order $1 / \hbar$. The field strengths in the core of the flux tubes we study are of order $\hbar^{0}$.

Secondly, we do not understand how $g_{s}$ emerges as the closed string coupling in our scenario. In our solutions, $g_{s}$ scales out of the effective coupling of the open string degrees of freedom. It is not clear how the coupling between flux tubes can be $g_{s}$. With more control over this system, we would be able to measure this coupling by studying the probability for two flux tubes to intersect and rejoin as in Fig. 2.

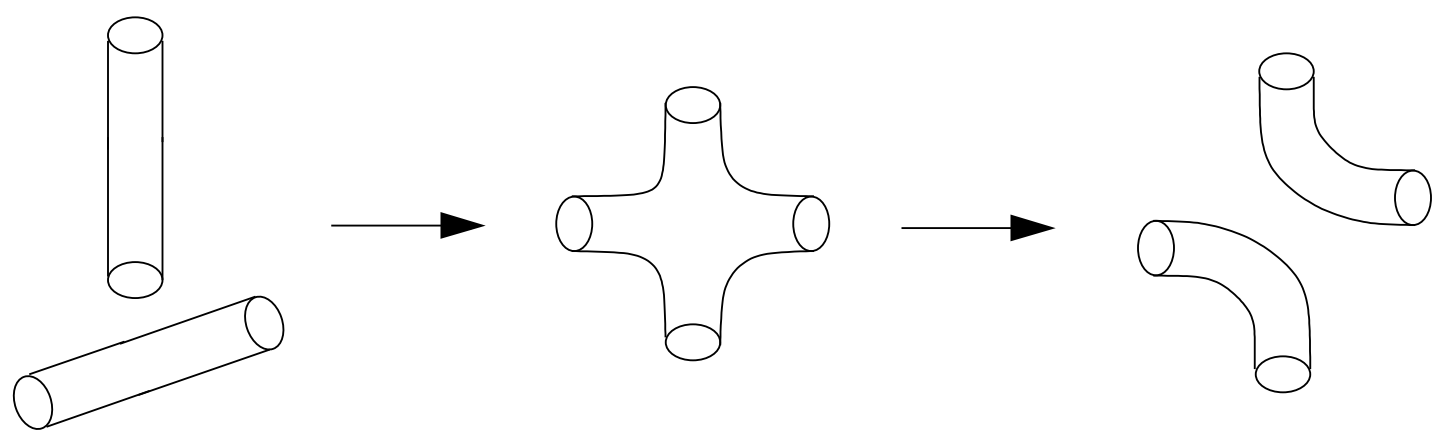

Figure 2. Fluxtube interaction.

Finally, a more "mundane" project suggested by sec. 2 is to better understand the relationship between different presentations of open string field theory. The cubic SFT is tied in a relatively intuitive way to worldsheet geometry - the moduli of a collection of vertices and propagators with a given topology cover the moduli of Riemann surfaces with that topology $[\mathbb{8}]$. The quantum version of BSFT is not well understood. In view of its computational power and conceptual elegance in understanding classical tachyon condensation, this picture deserves to be developed further.

\section{Acknowledgements}

8 We would like to thank L. Susskind for stressing this point. 
We would like to thank O. Bergman, M. Douglas, D. Gross, J. Harvey, K. Hori, P. Kraus, D. Kutasov, E. Martinec, J. McGreevy, G. Moore, A. Sen, E. Silverstein, L. Susskind, W. Taylor, N. Toumbas, N. Warner and P. Yi for useful conversations and comments. Some of this work was carried out at the Aspen Center for Physics "String Dualities" workshop. A.L. would also like to thank the Rutgers High Energy Physics Group for their hospitality while this work was initiated. M.K. was supported in part by Stanford University. A.L. was supported in part by the DOE under contract DE-AC03-76SF00515, and in part by a DOE OJI grant awarded to E. Silverstein. S.S. was supported in part by NSF grant PHY-9870115.

\section{Appendix A. Field redefinitions}

We would like to understand what field redefinitions could relate the variables natural to the cubic and "background-independent" string field theories of [7,48].

As a guide, we analyze the gauge invariance of the effective action. The gauge transformations of the string fields depends on the choice of coordinates on field space, which will depend on the worldsheet regulator used to define the propagator and interactions (c.f. 65]). We can study the gauge symmetries of the effective action to get some insight into the field redefinitions one needs to relate the calculations in [15, 16, 38, 39] to the calculations in [50,40,36]. In general these field redefinitions will be complicated and nonlocal and involve all of the string fields. We will study local transformations of the tachyon and gauge field only, which should suffice for nearly static configurations after integrating out the auxilliary fields.

Let $\tilde{\phi}, \tilde{A}$ be the tachyon and gauge field in the cubic open string field theory, where $\tilde{\phi}=0$ is the open string vacuum. At $\tilde{\phi}=0$, the $U(1)$ gauge transformation is [72, 63]:

$$
\begin{gathered}
\delta \tilde{\phi}=-\tilde{A}^{\mu} \partial_{\mu} \Lambda+\ldots \\
\delta t A_{\mu}=\partial_{\mu} \Lambda+\ldots
\end{gathered}
$$

where $\Lambda$ is the gauge parameter. It is already clear that the gauge transformations of $\tilde{\phi}, \tilde{A}$ are nontrivial. Away from $\tilde{\phi}=0$, the transformations will change. We will assume a more general form good far from the open string vacuum:

$$
\begin{array}{r}
\delta \tilde{\phi}=-f(\tilde{\phi}) \tilde{A}_{\mu} \partial_{\mu} \Lambda \ldots \\
\delta \tilde{A}_{\mu}=g(\tilde{\phi}) \partial_{\mu} \Lambda .
\end{array}
$$


Let us assume that the gauge transformations acting on the BSFT variables $\phi, A$ are of the standard form:

$$
\begin{aligned}
& \delta \phi=0 \\
& \delta A=\partial_{\mu} \Lambda,
\end{aligned}
$$

Under these gauge transformations, the potential energy $V(\tilde{\phi})$ is not gauge invariant. Consider small fluctuations of the gauge field. To lowest order in $\Lambda$ and $\tilde{A}$, the gaugeinvariant completion is:

$$
\mathcal{V}(\tilde{\phi}, \tilde{A})=V(\tilde{\phi})+\frac{1}{2} \frac{f}{g} \partial_{\tilde{\phi}} V \tilde{A}^{2}+\ldots
$$

The field redefinitions required to relate the gauge invariances (A.2), (A.3) at this lowest order in $\tilde{A}$ are:

$$
\begin{aligned}
\phi & =\tilde{\phi}+\frac{1}{2} \frac{f}{g}(\tilde{\phi}) \tilde{A}^{2} \\
A & =\frac{\tilde{A}}{g(\phi)}
\end{aligned}
$$

At the minimum of $V(\tilde{\phi})$, the mass term vanishes unless $g(\tilde{\phi})$ has a zero at the same order as $\partial_{\tilde{\phi}} V$. In this case, the field redefinitions are singular precisely at the closed string vacuum. It is not clear if one set of coordinates is more natural.

We can turn this story around and begin with the variables $\phi, A . V(\phi)$ has been calculated exactly (at tree level) in BSFT. If we expand $V(\phi)$ in $\tilde{A}^{2}$ we will find the same mass term as above. We will also find that higher-order terms in $\tilde{A}^{2}$ will diverge, because of the pole in (A.5) and because of the divergences in higher derivatives of (2.3). The small fluctuation expansion breaks down badly. In order to understand the relationship between the various calculations, we need a better handle on interaction terms (and on higher-derivative terms) in the cubic string field theory. 


\section{References}

[1] T. Banks, W. Fischler, S. Shenker and L. Susskind, "M theory as a matrix model: a conjecture," Phys. Rev. D55 (1997) 5112; hep-th/9610043.

[2] J. Maldacena, "The large-N limit of superconformal field theories and supergravity," Adv. Theor. Math. Phys. 2 (1998) 231; hep-th/9711200.

[3] For discussions of this speculation see the talk by S. Shenker at http://quark.theory.caltech.edu/people/rahmfeld/Shenker/fs1.html and D. Bigatti and L. Susskind, "TASI Lectures On The Holographic Principle," hep-th/0002044.

[4] B. Zwiebach, "Closed string field theory: quantum action and the B-V master equation," Nucl. Phys. B390 (1993) 33; hep-th/9206084.

[5] J. Polchinski, "What is string theory?" in Fluctuating Geometries in Statistical Mechanics and Field Theory, F. David and P. Ginsparg, eds.

[6] S. Shenker, "The strength of nonperturbative effects in string theory"; in Cargese 1990: Proceedings, Random Surfaces and Quantum Gravity O. Alvarez, E. Marinari and P. Windey, eds. (Plenum, NY, 1990) 191.

[7] E. Witten, "Noncommutative geometry and string field theory," Nucl. Phys. B268 (1986) 253.

[8] S.B. Giddings, E. Martinec and E. Witten, "Modular invariance in string field theory," Phys. Lett. B176 (1986) 362.

[9] N. Berkovits, "Super-Poincaré invariant superstring field theory," Nucl. Phys. B450 (1995) 90; erratum in ibid., B459 (1996) 439; hep-th/9503099.

[10] N. Berkovits, "A new approach to superstring field theory," Fortsch. Phys. 48 (2000) 31 ; hep-th/9912121.

[11] S.B. Giddings and E. Martinec, "Conformal geometry and string field theory," Nucl. Phys. B278 (1986) 91.

[12] A. Sen, "Tachyon condensation on the brane anti-brane system," J. High Energy Phys. 9808 (1998) 012; hep-th/9805170.

[13] A. Sen, "Supersymmetric worldvolume action for non-BPS D-branes," , J. High Energy Phys. 9910 (1999) 008; hep-th/9909062.

[14] A. Sen, "Universality of the tachyon potential," J. High Energy Phys. 9912 (1999) 027; hep-th/9911116.

[15] A. Sen and B. Zwiebach, "Tachyon condensation in string field theory," J. High Energy Phys. 0003 (2000) 002; hep-th/9912249.

[16] N. Berkovits, A. Sen and B. Zwiebach, "Tachyon condensation in superstring field theory," Nucl. Phys. B587 (2000) 147; hep-th/0002211.

[17] K. Bardakci, "Dual models and spontaneous symmetry breaking," Nucl. Phys. B68 (1974) 331. 
[18] K. Bardakci and M.B. Halpern, "Explicit spontaneous breakdown in a dual model," Phys. Rev. D10 (1974) 4230.

[19] K. Bardakci and M.Bi. Halpern, "Explicit spontaneous symmetry breakdown in a dual model II: N point functions," Nucl. Phys. B96 (1975) 285.

[20] K. Bardakci, "Spontaneous symmetry breakdown in the standrad dual string model," Nucl. Phys. B133 (1978) 297.

[21] A. Sen, "SO(32) spinors of type I and other solutions on brane-anti-brane pair," J. High Energy Phys. 9809 (1998) 023; hep-th/9808141.

[22] A. Sen, "Descent relations among bosonic D-branes," Int. J. Mod. Phys. A14 (1999) 4061; hep-th/9902105.

[23] A. Sen, "Vortex pair creation on brane-anti-brane pair via marginal deformation," $J$. High Energy Phys. 0006 (2000) 010; hep-th/0003124.

[24] R. de Mello Koch, A. Jevicki, M. Mihailescu and R. Tatar, "Lumps and p-branes in open string field theory," Phys. Lett. B482 (2000) 249; hep-th/0003031.

[25] J.A. Harvey and P. Kraus, "D-branes as unstable lumps in bosonic open string field theory," J. High Energy Phys. 0004 (2000) 012; hep-th/0002117.

[26] N. Moeller, A. Sen and B. Zwiebach, "D-branes as tachyon lumps in string field theory," J. High Energy Phys. 0008 (2000) 039; hep-th/0005036.

[27] P. Kraus, J.A. Harvey, F. Larsen and E.J. Martinec, "D-branes and strings as noncommutative solitons," J. High Energy Phys. 0007 (2000) 042; hep-th/0005031.

[28] K. Dasgupta, S. Mukhi and G. Rajesh, "Noncommutative tachyons," J. High Energy Phys. 0006 (2000) 022; hep-th/0005006.

[29] P. Hořava, "Type IIA D-branes, K-theory and matrix theory," Adv. Theor. Math. Phys. 2 (1999) 1373; hep-th/9812135. See also the Strings '99 talk "M-theory, Mach's principle, and tachyon condensation on branes" at http://strings99.aeipotsdam.mpg.de/.

[30] E. Witten, "Overview of K-theory applied to strings"; hep-th/0007175.

[31] P. Yi, "Membranes from five-branes and fundamental strings from Dp-branes," Nucl. Phys. B550 (1999) 214; hep-th/9901169.

[32] O. Bergman, K. Hori and P. Yi, "Confinement on the brane," Nucl. Phys. B580 (2000) 289 ; hep-th/0002223.

[33] G. Gibbons, K. Hori and P. Yi, "String fluid from unstable D-branes"; hep-th/0009061.

[34] A. Sen, "Fundamental strings in open string theory at the tachyonic vacuum"; hepth/0010240.

[35] J.A. Harvey, D. Kutasov and E.J. Martinec, "On the relevance of tachyons;" hepth/0003101.

[36] D. Kutasov, M. Mariño and G. Moore, "Some exact results on tachyon condensation in string field theory", J. High Energy Phys. 0010 (2000) 045; hep-th/0009148. 
[37] A.A, Gerasimov and S.L. Shatashvili, "Stringy Higgs mechanism and the fate of open strings"; hep-th/0011009.

[38] J. David, "U(1) gauge invariance from open string field theory," J. High Energy Phys. 10 (2000) 017; hep-th/0005085.

[39] W. Taylor, "Mass generation from tachyon condensation for vector fields on D-branes;" hep-th/0008033.

[40] A. Gerasimov and S. Shatashvili, "On Exact Tachyon Potential in Open String Field Theory"; hep-th/0009103.

[41] D. Kutasov, M. Mariño and G. Moore, "Remarks on tachyon condensation in superstring field theory"; hep-th/0010108.

[42] N. Seiberg, L. Susskind and N. Toumbas, "Strings in background electric field, space/time noncommutativity and a new noncritical string theory," J. High Energy Phys. 0006 (2000) 021; hep-th/0005040.

[43] R. Gopakumar, J. Maldacena, S. Minwalla and A. Strominger, "S-duality and noncommutative gauge theory," J. High Energy Phys. 0006 (2000) 036; hep-th/0005048.

[44] G. Gibbons, K. Hori, and P. Yi, "String fluid from tachyon condensation"; hepth/0009061.

[45] L. Cornalba, "Tachyon condensation in large magnetic fields with background independent string field thery"; hep-th/0010021.

[46] K. Okuyama, "Noncommutative tachyon from background independent open string field theory"; hep-th/0010028.

[47] O. Andreev, "Some computations of partition functions and tachyon potentials in background independent off-shell string theory"; hep-th/0010218.

[48] E. Witten, "On background-independent open string field theory," Phys. Rev. D46 (1992) 5467; hep-th/9208027.

[49] R. Gopakumar, S. Minwalla and A. Strominger, "Symmetry restoration and tachyon conensation in open string theory"; hep-th/0007226.

[50] E. Witten, "Some computations in background-independent off-shell string theory," Phys. Rev. D47 (1993) 3405; hep-th/9210065.

[51] J. Minahan and B. Zwiebach, "Field theory models for tachyon and gauge field dynamics," J. High Energy Phys. 09 (2000) 029; hep-th/0008231.

[52] J. Minahan and B. Zwiebach, "Effective tachyon dynamics in superstring theory"; hep-th/0009246.

[53] K. Li and E. Witten, "Role of short distance behavior in off-shell open string field theory," Phys. Rev. D48 (1993) 8535; hep-th/9303067.

[54] S. Shatashvili, "Comment on the background independent open string field theory," Phys. Lett. B311 (1993) 83; hep-th/9303143.

[55] S. Shatashvili, "On the problems with background independence in string theory"; hep-th/9311177. 
[56] I. Affleck and A.W.W. Ludwig, "Universal noninteger 'ground state degeneracy' in critical quantum systems," Phys. Rev. Lett. 67 (1991) 161.

[57] P. Fendley, H. Saleur and N.P. Warner, "Exact solution of a massless scalar field with a relevant boundary interaction;", Nucl. Phys. B430 (1994) 577; hep-th/9406125.

[58] M. Douglas, D. Kabat, P. Pouliot and S. H. Shenker, "D-branes and short distances in string theory," Nucl. Phys. B485 (1997) 85; hep-th/9608024.

[59] S.B. Giddings, "The Veneziano amplitude from interacting string field theory," Nucl. Phys. B278 (1986) 242.

[60] S. Elitzur, E. Rabinovici and G. Sarkissian, "On least action D-branes," Nucl. Phys. B541 (1999) 246; hep-th/9807161.

[61] J.A. Harvey, S. Kachru, G. Moore and E. Silverstein, "Tension is dimension," J. High Energy Phys. 0003 (2000) 001; hep-th/9909072.

[62] J. Kogut and L. Susskind, "Vacuum polarization and the absence of free quarks in four dimensions," Phys. Rev. D9 (1974) 3501.

[63] V.A. Kostelecky and S. Samuel, "On a nonperturbative vacuum for the open bosonic string," Nucl. Phys. B336 (1990) 263.

[64] A. Sen and B. Zwiebach, "Large marginal deformations in string field theory," J. High Energy Phys. 0010 (2000) 009; hep-th/0007153.

[65] V.A. Kostelecky, M.J. Perry and R. Potting, "Off-shell structure of the string sigma model," Phys. Rev. Lett. (2000); hep-th/9912243.

[66] A. Sen, "Some issues in non-commutative tachyon condensation," J. High Energy Phys. 011 (2000) 035; hep-th/0009038.

[67] N. Seiberg and E. Witten, "String theory and noncommutative geometry," J. High Energy Phys. 9909 (1999) 032; hep-th/9908142.

[68] C.P. Burgess, "Open string instability in background electric fields," Nucl. Phys. B294 (1987) 427.

[69] A. Sen, private correspondence.

[70] W. Taylor, talk given at Stanford University, and private discussions.

[71] D. Ghoshal and A. Sen, "Tachyon condensation and brane descent relations in p-adic string theory," Nucl. Phys. B584 (2000) 300; hep-th/0003278.

[72] W. Siegel and B. Zwiebach, "Gauge string fields," Nucl. Phys. B263 (1986) 105. 